A N N A L E S Annales de Bretagne et des Pays de l'Ouest

Anjou. Maine. Poitou-Charente. Touraine

$111-2 \mid 2004$

Varia

\title{
Vivre au-delà des ponts à Angers (1380-1499)
}

Étude de la société angevine à partir des censiers de l'abbaye du Ronceray

Anne-Claire Mérand

\section{OpenEdition}

9 Journals

Édition électronique

URL : http://journals.openedition.org/abpo/1277

DOI : $10.4000 /$ abpo. 1277

ISBN : 978-2-7535-1494-2

ISSN : 2108-6443

Éditeur

Presses universitaires de Rennes

Édition imprimée

Date de publication : 20 juillet 2004

Pagination : 7-28

ISBN : 978-2-7535-0031-0

ISSN : 0399-0826

\section{Référence électronique}

Anne-Claire Mérand, "Vivre au-delà des ponts à Angers (1380-1499) ", Annales de Bretagne et des Pays de l'Ouest [En ligne], 111-2 | 2004, mis en ligne le 20 juillet 2006, consulté le 01 mai 2019. URL : http:// journals.openedition.org/abpo/1277 ; DOI : 10.4000/abpo.1277 


\title{
Vivre au-delà des ponts à Angers (1380-1499)

\author{
Étude de la société angevine \\ à partir des censiers de l'abbaye du Ronceray
}

\author{
Anne-Claire MÉRAND \\ Professeur certifié d'Histoire-Géographie \\ Lycée Rotrou, Dreux
}

\begin{abstract}
À la fin du Moyen Âge, à l'instar de ce qui se passe encore aujourd'hui, un soin particulier est porté aux documents fiscaux : terriers, censiers, baux... En effet, comme le souligne Robert Fossier, « dans un monde où la fortune et la puissance sont essentiellement d'origine foncière ${ }^{1}$ ", il apparaît très important aux yeux des seigneurs, tant laïcs qu'ecclésiastiques, d'établir un tableau de leurs biens et revenus, notamment en périodes de troubles ou de contestations. Les censiers, sur la nature desquels nous reviendrons, constituent ainsi des documents particulièrement précieux, tant pour leurs contemporains que pour les historiens auxquels ils procurent des renseignements tout aussi riches sur les hommes et les biens qu'ils envisagent. De ce point de vue, l'étude des censiers de l'abbaye du Ronceray - monastère féminin de la ville d'Angers - s'avère particulièrement intéressante. De fait, les livres fonciers de cet établissement ecclésiastique ont été bien conservés, à tel point que nous disposons encore à ce jour d'un riche ensemble de trois censiers des XIV et XV siècles, dont deux ont fait l'objet d'une transcription ${ }^{2}$. L'étude et la comparaison de ces sources fiscales nous permettent d'esquisser le tableau d'une partie de la société angevine dans le dernier siècle du Moyen Âge.
\end{abstract}

1. FossiER, Robert, Polyptyques et censiers, Typologie des sources du Moyen Âge occidental, fascicule $\mathrm{n}^{\circ} 28$, Turnhout, 1978.

2. La transcription indexée du censier du Ronceray de 1385-1386 (Bibl. mun. Angers, ms. 852 [764] 119 folios) est présente dans mon mémoire de maîtrise : MÉRAND, AnneClaire, L'abbaye du Ronceray dans la ville d'Angers à la fin du Moyen Âge (vers 1380-1499). Affirmation d'une communauté de femmes en milieu urbain, Université d'Angers, 2001. Quant à celle du censier de 1460-1486 (Bibl. mun. Angers ms. 853 [765] 131 folios), elle est publiée dans le mémoire de maîtrise de ComBE, Myriam, Le quartier de la Doutre à Angers, à partir du censier du Ronceray de 1460. Implantation des élites, Université catholique d'Angers, 1999. 
Les livres fonciers sont des documents familiers aux médiévistes. De nombreuses études leur ont ainsi été consacrées. Longtemps considérés comme des sources d'histoire économique, nombre d'entre eux font aujourd'hui l'objet d'une relecture destinée à mettre en lumière l'ensemble des données sociales qu'ils recèlent. Si les livres fonciers évoquent généralement la terre, les hommes et les rapports seigneuriaux, en consignant sous forme de listes nominatives l'état des redevances et charges dues à un seigneur, la portée proprement fiscale de certains d'entre eux nous incite à parler de " censiers" - autrement dit recueil des droits seigneuriaux portant sur la terre - plutôt que de terriers ou encore de polyptyques. Cette dimension fiscale, doublée d'une volonté de conférer à ce type de document une valeur probatoire et juridique accrue, est d'ailleurs clairement réaffirmée dans l'intitulé et le préambule des censiers ${ }^{3}$ de l'abbaye du Ronceray.

D’obédience bénédictine, l'abbaye du Ronceray est, à la fin du Moyen Âge, l'un des trois établissements réguliers de moniales que compte le diocèse d'Angers et le seul de cette même ville. C'est également le plus prestigieux, du fait de la noblesse et du nombre de ses religieuses ${ }^{4}$. Fondée au $\mathrm{XI}^{\mathrm{e}}$ siècle par le comte d'Anjou Foulque Nerra et sa femme Hildegarde, sur la rive droite de la Maine, l'abbaye du Ronceray a joué un grand rôle dans le développement de la cité andégave au-delà des ponts ainsi que dans le peuplement rapide de ce côté de la rivière. Partant, à la fin du Moyen Âge, les religieuses du Ronceray dominent-elles, outre de nombreux prieurés extra-urbains ${ }^{5}$, toute la Doutre ${ }^{6}$ ainsi que des biens épars dans l'ensemble de la ville d'Angers ${ }^{7}$. Le fonctionnement d'un tel établissement religieux nécessite d'importantes sources de revenus. En effet, les dépenses y sont nombreuses : entretien des bénédictines (nourriture, habillement...), restauration des bâtiments, rétribution des serviteurs, procureurs et receveurs de l'abbaye, coût des procédures judiciaires... Si une partie des reve-

3. Voir annexe.

4. Le procès verbal de l'élection de l'abbesse Catherine de Tonnerre en 1499 (Bibl. nat. de France, Housseau XII, pièce 7876), atteste la présence de 23 religieuses auxquelles il faut ajouter les novices, qui n'étaient pas autorisées à voter pour choisir la nouvelle abbesse. Au vu des informations dont nous disposons, il semble raisonnable de penser qu'une bonne vingtaine de moniales, issues des plus grandes familles angevines, composaient le chapitre du Ronceray à cette époque.

5. L'abbaye du Ronceray possède sept prieurés extra-urbains : Avénières (Mayenne, arr. Laval, cant. Laval), Saint-Lambert-du-Lattay (Maine-et-Loire, arr. Angers, cant. Thouarcé), Seiches (Maine-et-Loire, arr. Angers, cant. Seiches-sur-le-Loir), Courthamon (non localisé), le Bourg-des-Moutiers (Loire-Atlantique, arr. Saint-Nazaire, cant. Bourgneufen-Retz, comm. Les Moutiers-en-Retz), le Plessis-aux-Nonnains (Maine-et-Loire, arr. Segré, cant. Châteauneuf-sur-Sarthe, comm. Cherré) et Saint-Christophe de Mareil (Sarthe, arr. La Flèche, cant. La Flèche, comm. Mareil-sur-Loire).

6. Nom donné au quartier d'Outre-Maine, autrement dit sur la rive droite de celle-ci.

7. Quelques mentions de biens situés à la fontaine Saint-Maimbeuf, dans la paroisse de Saint-Laud (Voir annexes, $\mathrm{f}^{\circ}$ 51), rue Baudrière (Arch. dép. de Maine-et-Loire, $254 \mathrm{H}$ 471) ou encore à Pierre-Lize, en la paroisse de Saint-Michel-du-Tertre (Arch. dép. de Maineet-Loire, $254 \mathrm{H}$ 511), apparaissent dans les censiers et les baux de l'abbaye. 
nus du monastère provient des pensions apportées par les moniales ainsi que des oblations faites par les paroissiens de l'église qui en dépend, ces ressources s'avèrent insuffisantes pour couvrir l'ensemble des dépenses de l'abbaye. De fait, les religieuses tirent l'essentiel de leurs revenus du grand fief qui leur fut octroyé par le comte d'Anjou au XI ${ }^{\mathrm{e}}$ siècle.

\section{Délimitation du " grand fief " du Ronceray ${ }^{8}$}

Le fief urbain de l'abbaye se situe presque exclusivement sur la rive droite de la Maine, dans le quartier de la Doutre. Pour reconstituer le patrimoine urbain de l'abbaye du Ronceray à la fin du Moyen Âge, nous disposons de l'étude réalisée à ce sujet par Myriam Combe ${ }^{9}$ qui propose une restitution plus ou moins fidèle du fief de l'abbaye à la fin du $\mathrm{XV}^{\mathrm{e}}$ siècle (1460-1486). Si cette reconstitution nous offre une idée globale de ce que pouvaient être les possessions des bénédictines à Angers à cette époque, l'utilisation du plan que nous reproduisons ci-après s'accompagne cependant de quelques réserves (Plan 1).

Il semble, de fait, important de préciser que celui-ci a été réalisé à partir du cadastre napoléonien de 1840. En conséquence, et même si la configuration du quartier de la Doutre n'a que peu évolué par rapport à d'autres parties de la ville, l'agencement des parcelles ainsi que celui des rues a incontestablement changé en près de quatre cents ans ${ }^{10}$. En outre, cette étude ne repose que sur l'exploitation du censier du Ronceray de 14601486. Aucun censier antérieur n'a en effet été pris en considération pour la restitution du fief de l'abbaye. Il est à noter par ailleurs que les possessions du Ronceray s'avèrent d'autant plus difficiles à déterminer avec précision que certaines parcelles appartiennent également à l'hôpital Saint-Jean, dont le fief se trouve souvent imbriqué dans celui des moniales. De plus, la structure des trois censiers que nous conservons pour cette époque $(1385,1424-$ 1426 et 1460-1486) ne facilite pas le travail de localisation. En effet, ceux-ci sont organisés par termes (Noël, Saint-Jean-Baptiste, Pâques ou encore l'Angevine), par rue et enfin par personne. Aussi Hervé Le Clerc, censier de l'abbaye en 1385, de même que son homologue Gervaise Davaines, un siècle plus tard, ne suivent-ils pas les biens un à un en remontant ou descendant une rue, mais préfèrent évoquer toutes les possessions d'une même personne dans la rue, voire dans les rues attenantes. Enfin, loin d'être figé, le fief et les possessions des religieuses évoluent tout au long du

8. Avant de nous intéresser au temporel urbain de l'abbaye, il nous a semblé important de resituer ce fief dans un cadre plus large - l'ensemble des biens de l'abbaye en Anjou - afin de mieux réaliser l'ampleur de ces possessions et de souligner le fait que, malgré son importance déjà considérable dans la ville d'Angers, comme nous allons le voir, le fief du Ronceray ne se limite pas à ce seul espace géographique.

9. ComBe, Myriam, Le quartier de la Doutre..., op. cit.

10. Ainsi, nombre de rues de la fin du Xve siècle ont-elles disparu en 1840 : rue du PontLevay, rue de la Fôret, rue de la Chêvrerie, rue de la Couldre, rue de Corne-de-Cerf..., dans ComBE, Myriam, Le quartier de la Doutre..., op. cit., p. 35. 
$\mathrm{XV}^{\mathrm{e}}$ siècle, comme en témoignent les censiers, les procédures, les baux ainsi que les contrats d'acquêts. Cependant, ces acquisitions ne modifient que très peu l'allure générale du fief des bénédictines qui reste sensiblement le même dans le dernier siècle du Moyen Âge.

Plan 1 - Le fief du Ronceray à la fin du Moyen Âge

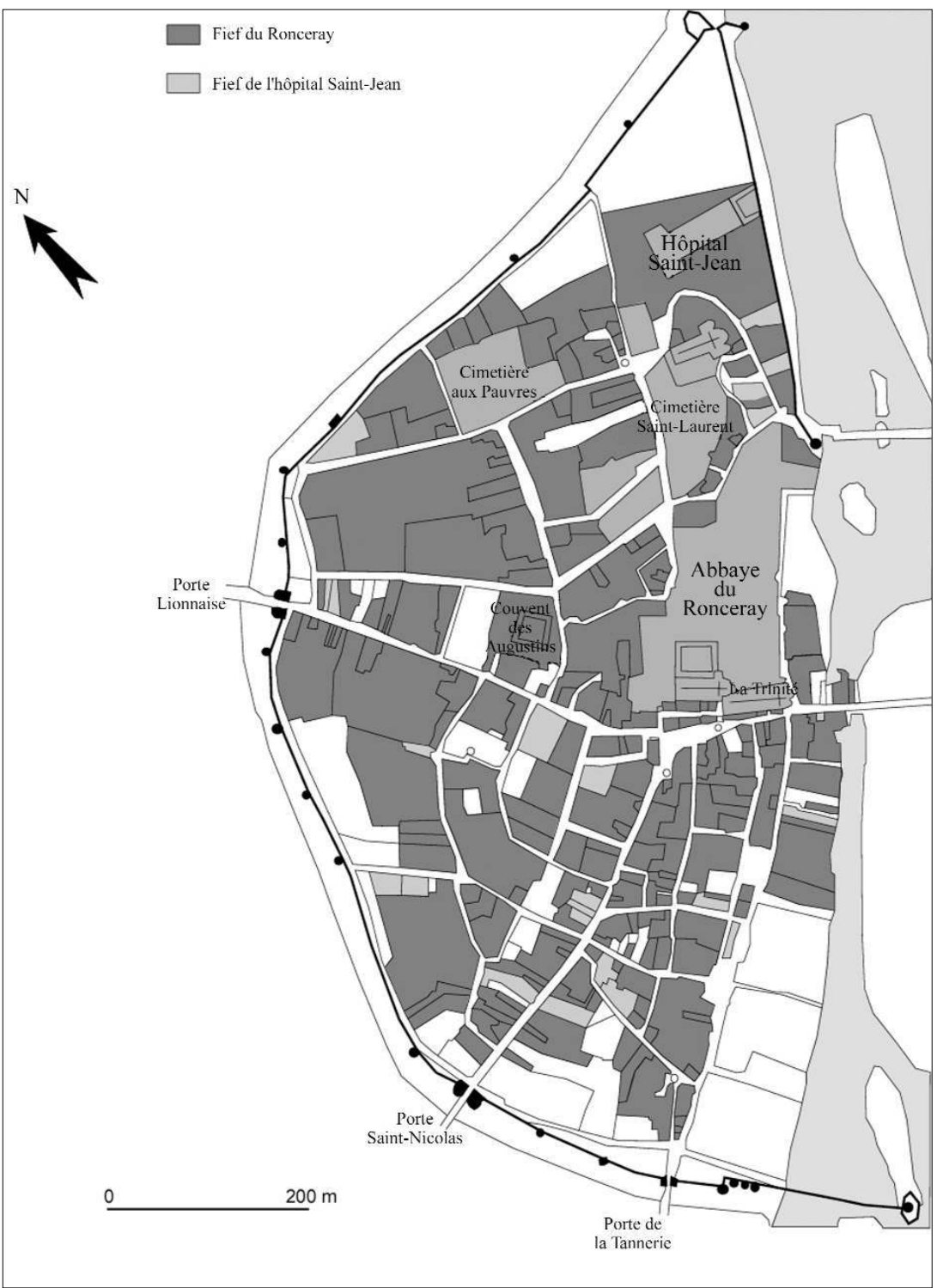


Cette carte de la Doutre ne doit donc pas être considérée comme une représentation exacte des possessions de l'abbaye, ce qu'elle ne prétend d'ailleurs pas être. Elle a cependant le mérite d'être la première - et la seule - tentative de restitution de ce patrimoine urbain et en fournit indubitablement une bonne idée générale.

L'organisation topographique de la Doutre est à mettre en lien direct avec son histoire. En effet, comme nous avons déjà eu l'occasion de le rappeler, ce quartier naît véritablement et se développe en même temps que l'abbaye $\mathrm{au} \mathrm{XI}^{\mathrm{e}}$ siècle. Situé sur la rive droite de la Maine - axe commercial il est cependant relié par plusieurs ponts au reste de la ville avec laquelle il entretient de nombreuses relations. Ces éléments ne sont pas sans incidences sur la structure du fief du Ronceray. Celui-ci s'organise en effet autour de trois pôles.

Les différents établissements ecclésiastiques de ce quartier constituent, à n'en pas douter, le principal de ces pôles, avec à leur tête l'abbaye et l'église de la Trinité. Seule la localisation du Ronceray nous est connue. Situé en bord de Maine, ce vaste ensemble religieux fait face à la cathédrale - si l'on suit l'axe du grand pont d'Angers ${ }^{11}$ - dont elle semble alors être plus ou moins le pendant sur la rive droite. Elle y occupe de fait une place prépondérante. L'hôpital Saint-Jean constitue un autre élément structurant de cette partie de la ville du fait de l'ancienneté de son implantation - à la fin du XII ${ }^{\mathrm{e}}$ siècle - de l'importance de son fief, qui se mêle à maintes reprises à celui de l'abbaye. Il en va de même pour le couvent mendiant des Augustins également situé dans la Doutre.

Outre les établissements ecclésiastiques, trois grandes rues commerçantes traversent le fief du Ronceray de part en part : la rue Saint-Nicolas, la rue Lionnaise et la grand-rue du Tertre. Celles-ci partent en effet de l'abbaye et se terminent au rempart - " les murs de la ville " - édifié au XIII ${ }^{\mathrm{e}}$ siècle à la demande de Blanche de Castille. Elles tirent leurs noms des lieux vers lesquelles elles mènent : l'abbaye Saint-Nicolas, le Lion-d'Angers et le Tertre Saint-Laurent.

Enfin, un élément naturel d'importance, la Maine, concourt également à l'organisation de la Doutre. En effet, les ponts qui la traversent sont des lieux de passage et de commerce privilégiés.

L'importance de tous les éléments que nous venons d'évoquer se retrouve à travers la toponymie. Certaines rues portent ainsi le nom d'établissements religieux (rue Notre-Dame), d'éléments naturels (rue de la Petite-Arche) ou d'activités économiques (la Boucherie, la Pâtisserie, la Tannerie). En dehors des trois artères que nous avons précédemment mentionnées, le fief du Ronceray correspond parfaitement au topos de la ville médiévale. Il est en effet entièrement quadrillé par de petites rues qui s'apparentent davantage à des venelles.

11. Le Grand Pont correspond aujourd'hui au pont de Verdun. 


\section{Les différents revenus issus du temporel}

Les bénédictines du Ronceray disposent tout au long de leur existence d'une grande variété de revenus du fait de leur fonction religieuse (dîmes, oblations) aussi bien que de leur qualité de seigneur.

Deux types de ressources tirées du temporel urbain, le cens et la rente, occupent incontestablement une place prépondérante dans les rentrées d'argent de l'abbaye. Bien différenciées dans la plupart des sources du $\mathrm{XV}^{\mathrm{e}}$ siècle, ces deux redevances sont le plus souvent confondues dans les actes émanant du monastère. Une distinction doit pourtant être établie entre ces deux notions. Selon la coutume d'Anjou, "le cens est la pencion et devoir qui est poié en signe de subgection à aucun seigneur de fié sur aucun heritage qui est tenu de luy ${ }^{12}$ ". En principe fixe et perpétuelle, cette redevance compte donc au nombre des droits féodaux des seigneurs fonciers, le cens portant sur la terre et non sur le bien, contrairement à ce que pourrait laisser entendre la présentation des censiers. Il est à noter en effet que si la nature des biens y est mentionnée, celle-ci n'a aucune incidence sur le montant du cens. Elle sert uniquement à localiser la parcelle accensée. La rente s'apparente quant à elle davantage à un loyer. Portant sur un bien, elle est versée au propriétaire et non au seigneur.

Six grands types de biens apparaissent de façon récurrente dans les sources concernant l'abbaye du Ronceray (Tableau 1). Le plus répandu est incontestablement la maison, qui constitue près de $50 \%$ des biens identifiés. Aucune indication ne nous est donnée quant à la dimension de ces habitations. Leur localisation, de même que celle des espaces - bâtis ou non - qui l'entourent, est en revanche presque toujours précisée. Il est ainsi fréquent de rencontrer dans les censiers une maison associée à d'autres types de biens, à commencer par les jardins. Ces espaces non-bâtis apparaissent de deux façons dans les censiers. Tout d'abord sous la simple dénomination jardins, mais aussi à travers l'expression courtil. Ce terme désigne en effet une maison avec cour et jardin. Il est en outre frappant de constater que les espaces non-bâtis comme les jardins et les places s'avèrent particulièrement nombreux dans la Doutre par rapport aux fiefs situés sur la rive gauche de la Maine. Cela s'explique par le fait que, bien qu'ayant connu un essor rapide à partir du XII ${ }^{\mathrm{e}}$ siècle, cette partie de la ville s'est développée beaucoup plus tardivement que la rive gauche. En outre, le fief du Ronceray est le plus vaste de la ville d'Angers. Cet état de fait n'est certainement pas étranger aux nombreuses " appartenances " qui accompagnent les maisons et courtils. De même, le nombre important d'hébergements, hôtels et manoirs - vastes habitations pourvues de multiples appartenances - est sans doute à mettre en lien direct avec l'espace disponible dans la Doutre, même si le facteur social n'est pas à négliger. En effet, nombreux sont les ecclésiastiques, les seigneurs et les bourgeois à

12. Beautemps-BeauPrÉ, Louis-Jean, Coutumes et institutions de l'Anjou et du Maine antérieures au XVI siècle, première partie, Coutumes et styles, Paris, 1877-1883, art. 1486. 
détenir des biens sur le fief du Ronceray. La présence d'une soixantaine d'appentis en moyenne durant tout le $\mathrm{XV}^{\mathrm{e}}$ siècle tient quant à elle à la longueur du rempart qui encercle alors une partie du fief des religieuses depuis près de deux siècles. " Demi-combles en auvent, à un seul égout, appuyés à une muraille et portés par des piliers ${ }^{13}$ ", les appentis se multiplient aussi bien le long des murs de la ville que de ceux de l'abbaye.

Tableau 1 - Les différents types de biens situés sur le fief du Ronceray

\begin{tabular}{|l|c|c|c|}
\hline & $1385-1386$ & $1424-1426$ & $1460-1486$ \\
\hline Hébergements, manoirs, hôtels & 41 & 27 & 26 \\
Maisons & 381 & 417 & 418 \\
Appentis & 47 & 69 & 69 \\
Courtils et jardins & 56 & 190 & 224 \\
Fours & 32 & 22 & 26 \\
Étals & 37 & 21 & 17 \\
Places & 17 & 47 & 26 \\
Total & 611 & 793 & 806 \\
\hline
\end{tabular}

L'activité économique des habitants de la Doutre s'avère également déterminante dans la construction de deux autres types de biens : les fours et les étals. Leur forte proportion dans cette partie de la ville est liée au travail des pâtissiers, boulangers, bouchers et forgerons, fortement représentés sur le fief de l'abbaye. Remarquons enfin qu'hormis les courtils et les jardins, le nombre de biens évolue peu en l'espace de cent ans. Qu'en est-il de leur état?

Nombreuses sont les mentions faisant référence à l'état des biens à la fin du XIV e siècle. Il apparaît ainsi que de multiples immeubles, maisons ou hébergements, sont alors " en ruyne " ou nécessitent des réparations. Cet état de fait nous est connu grâce à une rubrique du censier de 1385 traitant des " biens vacquants et en ruynes ${ }^{14}$ ". Il semble dès lors que les bénédictines aient constamment témoigné d'un réel souci de voir les biens entretenus. Cette préoccupation est loin d'être désintéressée; les moniales affirment d'ailleurs clairement leurs intentions dans les baux. Ainsi leur motivation est-elle liée à des intérêts économiques. Elles obligent de fait les tenanciers à maintenir leurs biens en bon état afin " que ledit cens ne se puisse deperir ${ }^{15}$ ». Il est à noter d'ailleurs qu'un délai très précis leur est imposé pour effectuer les réparations promises. Celui-ci oscille généralement entre un et trois ans. On peut dès lors raisonnablement supposer que cette dernière clause, particulièrement contraignante, fut ajoutée afin d'obliger les contractants à tenir leurs engagements; ce qui tendrait donc à démontrer que les travaux n'étaient pas systématiquement effectués.

13. DRIDI, Nabil, L'abbaye Saint-Aubin dans la ville d'Angers (1250-1450), Université d'Angers, 1995, p. 136.

14. Bibl. mun. Angers, ms 852 (764), $\mathrm{f}^{\circ} 90$.

15. Arch. dép. de Maine-et-Loire, 254 H 34. 
Outre des réparations, les religieuses exigent aussi parfois des tenanciers l'édification de nouveaux biens. Aussi certains sont-ils tenus de construire une cheminée ${ }^{16}$, un puits ${ }^{17}$ ou encore " une bonne maison ${ }^{18}$ ". De fait, en 1427, Henry Rousseau, maçon, et Henriette sa femme s'engagent à faire édifier une maison sous un an ${ }^{19}$. Cette politique de restauration, voire de réhabilitation, transparaît dans les censiers où l'on trouve quelques références à des maisons neuves.

Si les mesures pour se prémunir contre les tenanciers récalcitrants foisonnent dans le dernier siècle du Moyen Âge, il est toutefois important de souligner que les moniales du Ronceray ne sont pas opposées à toute négociation. En effet, réparations et constructions ne sont pas systématiquement imposées aux tenanciers. Ceux-ci peuvent, il est vrai, obtenir une réduction du montant du cens en échange de la remise en état du bien qui leur est baillé. Ainsi, après avoir entrepris des travaux fort coûteux pour restaurer des maisons rue Lionnaise, Guillaume Le Commandeur négocie avec les bénédictines la baisse de moitié du montant de la rente qu'il leur doit, pour avoir les moyens de continuer la remise en état de ses biens. Le montant de la redevance est donc ramené à six livres tournois en janvier 1450 (n. s.) ${ }^{20}$.

Le montant des cens prélevés par les bénédictines du Ronceray sur leur " grand fief " nous est connu grâce aux trois précieux censiers encore conservés pour les $\mathrm{XIV}^{\mathrm{e}}$ et $\mathrm{XV}^{\mathrm{e}}$ siècles. Le calcul du cens moyen perçu par les moniales permet de faire un premier constat : le montant du cens a peu évolué en un siècle. En effet, à l'exception de la rue Notre-Dame, et surtout de la rue de la Pâtisserie, le niveau du cens est resté sensiblement identique dans les principales rues de la Doutre durant tout le dernier siècle du Moyen Âge. Il est toutefois important de rappeler que les séries de données dont nous disposons, aussi exceptionnelles soient-elles pour l'époque qui nous intéresse, ne sont pas toujours rigoureuses. Les trois censiers que nous avons utilisés ont en effet été tenus par trois receveurs différents. En outre, l'utilisation des statistiques en histoire médiévale doit toujours être considérée comme indicative et non interprétée comme un reflet exact de la réalité.

Par ailleurs, aussi utile cela puisse-t-il paraître, il nous est impossible de proposer un cens moyen pour l'ensemble du fief, les écarts types à la moyenne étant parfois très importants. En effet, si le montant des cens s'inscrit dans une fourchette assez étroite à l'intérieur d'une même rue, les différences entre ces dernières peuvent en revanche s'avérer considérables. Ainsi, les rues de la Tannerie, de Normandie ou encore de la Cuaterie ne sont-elles en moyenne assujetties qu'à des cens inférieurs à 200 deniers,

16. Arch. dép. de Maine-et-Loire, $254 \mathrm{H} 473$.

17. Arch. dép. de Maine-et-Loire, 254 H 527, pièce 58.

18. Arch. dép. de Maine-et-Loire, 254 H 505, 254 H 516 et 254 H 553.

19. Arch. dép. de Maine-et-Loire, 254 H 553.

20. Arch. dép. de Maine-et-Loire, 254 H 505. 
alors que les propriétaires de biens situés rue Notre-Dame et rue de la Bourgeoisie doivent acquitter des cens oscillant entre 600 et 1000 deniers.

Graphique 1 - Évolution du cens moyen en deniers prélevé dans les rues d'Angers par l'abbaye du Ronceray (1386-1486)

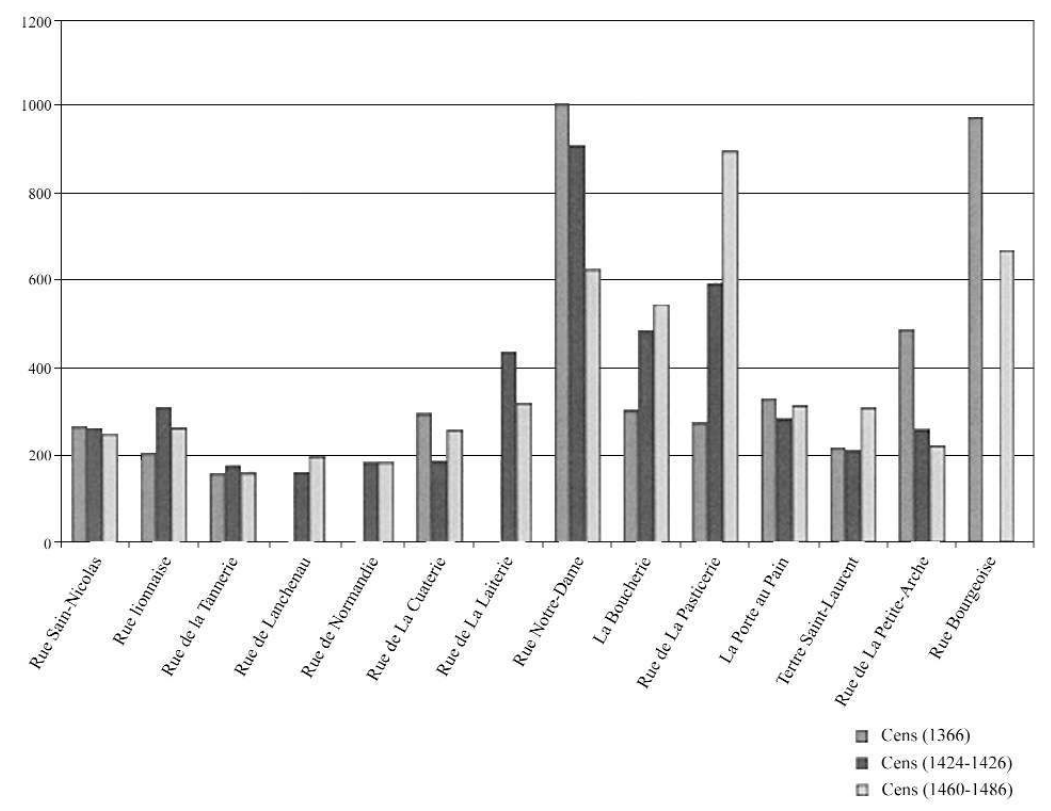

Ces écarts pour le moins conséquents sont à mettre en relation avec la localisation des rues elles-mêmes. En effet, une logique de prélèvement apparaît nettement si l'on replace les données moyennes obtenues pour chaque rue sur une carte de la Doutre (Plan 2). Trois constats s'imposent alors. Tout d'abord, le montant du cens est déterminé en fonction de la proximité de la parcelle par rapport au monastère et à l'église de la Trinité. Dès lors, plus les rues sont proches du Ronceray, plus le cens est élevé. Ainsi, la rue Notre-Dame, où le cens moyen est proche de 800 deniers dans le dernier siècle du Moyen Âge, longe-t-elle l'abbaye, comme son nom l'indique. Il en va de même pour la rue de la Pâtisserie ou encore la Boucherie où les cens prélevés avoisinent souvent 500 deniers. Cependant, dans ces trois rues, le cens moyen évolue considérablement et de façon contradictoire entre 1386 et 1460-1486. Le cens perçu rue Notre-Dame baisse en effet d'environ 40 \% en l'espace d'un siècle quand, dans le même temps, ceux de la Boucherie et de la Pâtisserie augmentent de plus ou moins 100 \%. Un premier facteur explicatif est à prendre en considération : la tenue des censiers. Comme nous avons déjà eu l'occasion de le souligner, les séries de chiffres dont nous disposons ne sont pas toujours construites de manière 
Plan 2-Les logiques de prélèvement du cens

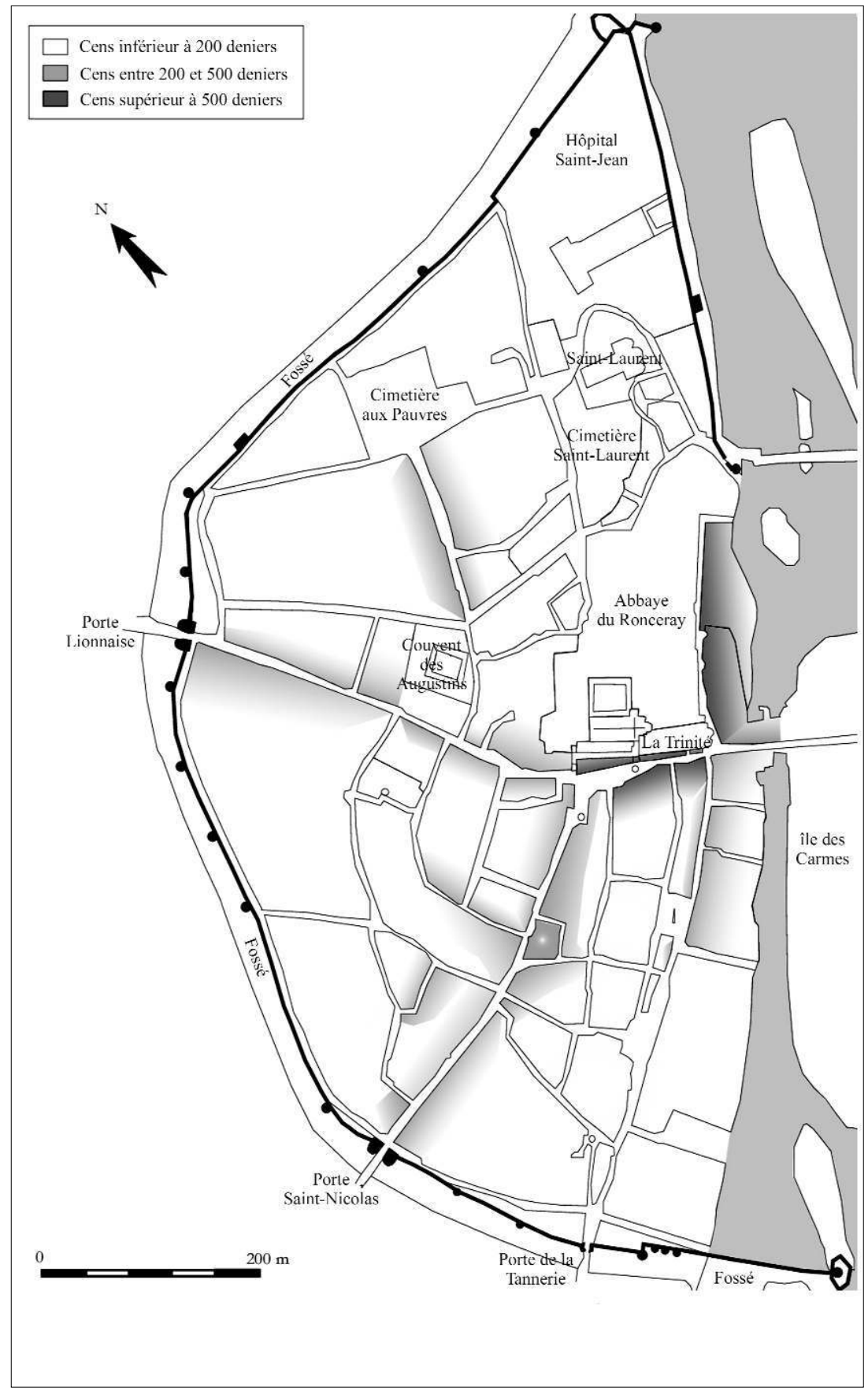


strictement identique entre 1386 et 1460-1486, suivant les receveurs. En outre, les cens moyens que nous avons représentés constituent, comme leur nom l'indique, une moyenne. Ainsi, sur des séries aussi brèves que celles conservées pour chacune des rues de la Doutre, le montant de chaque cens a une incidence importante sur la moyenne globale. Enfin, dans le cas de la Boucherie et de la Pâtisserie, le contexte politique et économique troublé explique en partie l'augmentation du cens dans ces rues marquées par des activités commerciales.

Ces deux derniers exemples mettent en lumière un autre critère déterminant dans la fixation du montant des cens : la fonction commerciale. De fait, les grandes rues marchandes - rue Saint-Nicolas, rue Lionnaise, la Porte au Pain et la rue du Tertre Saint-Laurent - sont soumises à des cens de 250 à 300 deniers. En outre, le cas de Jean Chappellain illustre parfaitement la logique d'imposition des bénédictines. Il obtient en effet de ces dernières la diminution du cens portant sur sa maison, rue de La Couldre - celui-ci étant rabattu de 4 à 2 livres - en alléguant que son bien se situe dans une rue foraine et non dans un lieu marchand ${ }^{21}$.

Si le montant des cens perçus sur les ponts s'inscrit indubitablement dans la logique commerciale que nous venons d'évoquer, ceux-ci constituent tout de même un cas à part. Du fait de leur situation privilégiée, ils sont l'objet des convoitises de tous les seigneurs - laïcs ou ecclésiastiques - de la ville (Tableau 2). L'abbaye du Ronceray se trouve ainsi confrontée aux velléités des autres établissements ecclésiastiques de la ville, à l'image de l'hôpital Saint-Jean ou encore des nombreuses collégiales comme SaintMartin. Elle s'avère donc extrêmement pointilleuse lors de l'octroi de baux portant sur la Petite Arche ou le Grand Pont. Le montant des cens est de ce fait très élevé : 800 deniers en moyenne rue Bourgeoise et 300 rue de $\mathrm{La}$ Petite-Arche. Il est à noter enfin que dans toutes les rues et les venelles qui ne répondent à aucun de ces trois critères les sommes perçues par les religieuses sont généralement inférieures à 120 deniers, quand elles sont seulement mentionnées dans les censiers ou les baux.

Cependant, contrairement à ce que l'on serait tenté de penser, les rues où le sol est le plus cher ne sont pas celles qui rapportent le plus à l'abbaye. Il ressort en effet de façon très nette que durant les cent ans qui retiennent notre attention, près d'un tiers des revenus des bénédictines proviennent des rues Saint-Nicolas et Lionnaise. Ceci s'explique essentiellement par la longueur de ces dernières. De fait, comme nous avons pu le constater, les cens perçus dans ces rues avoisinent les 300 deniers, ce qui est trois fois moins élevé que dans la rue de La Bourgeoisie. Toutefois, cette rue s'avère extrêmement courte par rapport aux deux artères précédemment citées. Aussi n'apparaît-elle même pas dans notre représentation graphique, tant la part qu'elle représente par rapport à l'ensemble des revenus du fief est faible - moins de deux pour cent. Elle compte ainsi au

21. Arch. dép. de Maine-et-Loire, 254 H 479. 
nombre de ces « rues diverses » où les religieuses du Ronceray perçoivent moins de dix livres sur l'ensemble de l'année.

Tableau 2 - Répartition des cens prélevés par le Ronceray dans la ville d'Angers

\begin{tabular}{|l|c|c|c|}
\hline & en $1385-1386$ & en $1424-1426$ & en $1460-1486$ \\
\hline Rue Saint-Nicolas & $16 \%$ & $20 \%$ & $15 \%$ \\
Rue Lionnaise & $10 \%$ & $13 \%$ & $14 \%$ \\
Rue de La Tannerie & $4 \%$ & $4 \%$ & $3 \%$ \\
Rue de Lanchenau & - & - & $2 \%$ \\
Rue de Normandie & - & - & $3 \%$ \\
Rue de La Cuaterie & $6 \%$ & $5 \%$ & $5 \%$ \\
Rue de La Laiterie & - & $3 \%$ & $5 \%$ \\
Rue Notre-Dame & $11 \%$ & $10 \%$ & $9 \%$ \\
La Boucherie & $5 \%$ & $4 \%$ & $4 \%$ \\
Rue de La Pasticerie & $4 \%$ & $3 \%$ & $5 \%$ \\
La Porte au Pain & $6 \%$ & $3 \%$ & $2 \%$ \\
Tertre Saint-Laurent & $5 \%$ & $5 \%$ & $4 \% \%$ \\
Rue de La Petite-Arche & $3 \%$ & $3 \%$ & $2 \%$ \\
Rue Bourgeoise & - & - & - \\
Joignant à la Trinité & $5 \%$ & - & - \\
Rue de Malemort & $2 \%$ & $3 \%$ & $4 \%$ \\
Rue des Raffoux & - & - & $2 \%$ \\
Rue de La Couldre & - & $20 \%$ & $15 \%$ \\
Rues diverses & $23 \%$ & & \\
\hline
\end{tabular}

Dès lors, quatre pôles principaux se dégagent. Aux deux grandes rues commerciales viennent s'ajouter la rue Notre-Dame et la grand rue du Tertre Saint-Laurent. Le nom de cette dernière est d'ailleurs significatif de sa longueur et donc du nombre de biens qui y sont concentrés. Le cas de la rue Notre-Dame s'apparente quant à lui davantage à celui de la rue SaintAubin, sur la rive gauche de la Maine. Son importance tient tout autant à sa longueur qu'au montant élevé des cens auxquels les tenanciers sont assujettis. Un dernier constat s'impose à la lecture des graphiques illustrant la répartition des cens prélevés par le Ronceray dans la ville d'Angers. Dans la Doutre, et contrairement au cas de Saint-Aubin que nous utilisons au titre de la comparaison, les revenus liés aux cens se répartissent de façon presque équivalente entre une dizaine de rues secondaires ${ }^{22}$. Tout au long du $\mathrm{XV}^{\mathrm{e}}$ siecle, ces rues représentent respectivement près de cinq pour cent des revenus du fief du Ronceray.

Le paiement des cens et rentes s'inscrit dans le calendrier des fêtes religieuses. Le nombre d'échéances s'avère des plus variables. En effet, certains tenanciers acquittent leurs redevances en une, deux, trois, voire quatre fois. Il semble que le nombre d'échéances soit lié au montant des

22. Rue de la Tannerie, de la Cuaterie, de la Pasticerie, la Boucherie ou encore la Porte au Pain. 
cens et rentes exigé par les bénédictines. Ainsi, plus il est élevé, plus les " contribuables " peuvent étaler leurs versements. Tenu de payer 840 deniers pour une maison et un four, rue de la Pâtisserie, Jean Duvivier règle son dû à quatre termes différents (Noël, Pâques, la Saint-Jean-Baptiste et Saint-Michel au Mont-Gargan), échelonnés sur l'ensemble de l'année, tous les trois mois environ ${ }^{23}$. Toutefois, la grande majorité des personnes assujetties à un cens ou une rente s'acquittent de leur dû en deux fois. Dans près de $80 \%$ des cas, le paiement s'effectue par moitié à Noël et à la SaintJean-Baptiste. Néanmoins, quelques rares tenanciers - environ $3 \%$ - règlent leur dû à Pâques et à la mi-août, ou à la Saint-Michel au Mont-Gargan. Dans tous les cas, un intervalle de six mois entre les deux termes retenus permet aux tenanciers d'amortir cette somme sur l'ensemble de l'année. Il est à noter cependant que la fête de l'Angevine (le 8 septembre) - jour de la nativité de la Vierge et de l'une des foires d'Angers - est également l'une des échéances les plus fréquemment retenues pour le paiement des cens. Néanmoins, au contraire de Noël et de la Saint-Jean-Baptiste, les montants qui y sont perçus s'avèrent minimes; tout au plus quelques dizaines de deniers. Aussi, les personnes redevables d'un faible cens choisissent-elles généralement cette date pour s'acquitter de la totalité de leur dû. Cette logique de versement n'est pas propre au Ronceray. On retrouve en effet les mêmes termes dans les mêmes proportions dans le cas de l'hôpital Saint-Jean ${ }^{24}$ et de l'abbaye Saint-Aubin d'Angers entre 1400 et $1450^{25}$. Le paiement des cens et rentes se fait à la " recepte de la Chambrerie ${ }^{26}$ ", autrement appelée " recepte de la Censerie ${ }^{27}$ ", ou encore " Hostel de la Censerie dudit moustier ${ }^{28}$ ". Situé dans la rue qui porte encore aujourd'hui son nom, cet Hôtel était attenant au monastère. Les tenanciers y payaient leur cens au niveau de la Porte du même nom. Ce règlement s'effectuait généralement en " monnaie courrant " - du fait du montant abordable exigé par les religieuses - comme en témoigne la grande majorité des baux ${ }^{29}$.

Afin de prémunir les seigneurs contre les mauvais payeurs, des clauses de garanties sont introduites dans les baux à partir du XIII siècle. Ces pratiques se développent et se complexifient encore dans les deux derniers siècles du Moyen Âge. Ainsi, au Xve siècle, le tenancier s'engage-t-il inéluctablement, devant témoins, à respecter les obligations prises envers les moniales, à savoir le paiement du cens aux échéances déterminées. Par la

23. Voir annexes, $\mathrm{f}^{\circ} 1 \mathrm{v}^{\circ}$.

24. Сначмот, Frédéric, L'hôpital Saint-Jean l'Évangéliste d'Angers (vers 1440-vers 1510). Typologie d'une crise hospitalière, de la fin de la guerre de Cent ans à la communalisation, Université d'Angers, 1996.

25. Dridi, Nabil, L'abbaye Saint-Aubin..., op. cit., p. 120-122.

26. Arch. dép. de Maine-et-Loire, 254 H 527, pièce 54.

27. Arch. dép. de Maine-et-Loire, $254 \mathrm{H} 32$ et 254 H 505.

28. Arch. dép. de Maine-et-Loire, 254 H 520.

29 . Il est à noter à ce propos que les monnaies en circulation à cette époque étaient des plus variées. On distingue en effet dans les baux et contrats d'acquêt : les royaux, les écus d'or, les écus d'or à la Couronne (valant 420 deniers), les écus d'or au Soleil (valant 423 deniers), les ducats (valant 450 deniers) et " Les francs or du coign du Roy ". 
signature du bail, il accepte que ses biens servent de garantie et renonce en outre à revenir sur ses engagements ou à intenter une procédure quelconque dans ce but, comme en témoigne la formule récurrente « oblige ledit [...], soy, ses hoirs, avecques touz et chacuns ses biens meubles et immeubles presents et à venir quelx qu'ilz soient. [...] Et tout ce que dessus est dit et divisé sans jamais venir encontre par applegement, contrapplegement, ne aucunement, en aucune manière, en est tenu ledit [...] par la foy et serment de son corps ". L'abbaye permet également aux tenanciers d'avoir recours à des garants à défaut de pouvoir engager leurs propres biens, caution insuffisante. Ainsi, lorsque le 7 janvier 1461 (n. s.), Pierre Cady se retrouve dans l'impossibilité de verser aux moniales du Ronceray les douze livres qu'il leur doit, ces dernières se retournent contre Jean de Souenne qui se voit dès lors dans l'obligation de payer cette somme. De ce fait, la grande majorité des tenanciers semblent respecter les échéances de paiement. Cependant, douze procédures intentées à cette époque par les bénédictines pour des questions d'arrérages subsistent encore dans les archives du Ronceray ${ }^{30}$. Cette proportion s'avère particulièrement importante lorsque l'on sait que, pour la même période, seule une procédure de ce type apparaît dans le fonds de l'abbaye Saint-Aubin d'Angers ${ }^{31}$. Les réclamations des religieuses portent le plus souvent sur quelques dizaines de livres. L'importance de ce montant est à mettre en relation avec le nombre d'années d'arrérages. En effet, ce chiffre oscille entre deux et vingt ans. Ainsi, la veuve de Gervaise Proces a-t-elle fait défaut de payer la rente de 6 livres tournois à laquelle elle était tenue pour une maison rue Baudrière, de 1401 à 1420. Un jugement est rendu en 1428, faisant apparaître qu'à cette date, le montant de ses arrérages s'élève à 61 livres, 18 sous, 4 deniers ${ }^{32}$.

Les raisons de ces défauts de paiement restent le plus souvent inconnues. Les tenanciers étaient-ils insolvables? Refusaient-ils purement et simplement de payer? Quelques éléments de compréhension nous sont néanmoins fournis par trois actes. Le premier met en lumière les difficultés rencontrées par la veuve de Gervaise Proces dont nous venons d'exposer le cas. Celle-ci allègue en effet pour sa défense que, depuis 1405, sa maison étant en ruine, les religieuses ont accepté de réduire le montant du cens à 4 livres, 10 sous, en échange de la remise en état de son bien; ce que nient les bénédictines. D'autres tenanciers, à l'image de Jeanne d'Ancenis, estiment quant à eux ne rien devoir, prétendant ne plus tenir les biens sujets à réclamation ${ }^{33}$. Certains vont d'ailleurs jusqu'à désigner un autre tenancier. Ainsi, Jean Mahé, poursuivi pour un peu plus de 5 livres d'arrérages, rejette-t-il la faute sur Guillaume Le Commandeur auquel les moniales auraient, selon lui, baillé une partie des possessions en question ${ }^{34}$. Enfin,

30. Ne sont pas prises en compte dans cette estimation les procédures opposant l'abbaye à d'autres établissements ecclésiastiques pour des problèmes d'arrérages.

31. DRIDI, Nabil, L'abbaye Saint-Aubin..., op. cit., p. 126-128.

32. Arch. dép. de Maine-et-Loire, 254 H 471.

33. Arch. dép. de Maine-et-Loire, 254 H 479.

34. Arch. dép. de Maine-et-Loire, 254 H 505. 
des problèmes se posent également du fait de l'imbrication des fiefs que nous avons déjà eu l'occasion de souligner. En février 1444 (n. s.), Guillaume Croière et sa femme cessent de verser leur cens aux religieuses du Ronceray, jugeant que leurs appartenances près de la Pierre Monconseil leur ont été baillées par les prieurs et frères de la Haie-aux-Bonshommes pour dix sous tournois de rente annuelle ${ }^{35}$.

Près de la moitié des tenanciers assignés en justice par l'abbaye sont des femmes veuves. On peut dès lors raisonnablement supposer que cellesci se trouvent dans l'impossibilité d'honorer les engagements pris du vivant de leur mari, une fois celui-ci décédé. Par ailleurs, tous ces modes de défense, rejetés en bloc par les religieuses dont les archives fiscales s'avèrent extrêmement précises pour l'époque, sont abandonnés par les tenanciers au cours des procès. La condamnation des mauvais payeurs consiste le plus souvent dans le remboursement des arrérages et dans l'obligation faite à ceux-ci de continuer à acquitter, régulièrement désormais, les sommes dues à l'abbaye.

Outre le simple remboursement, les religieuses disposent, comme tous les autres seigneurs, de nombreux moyens pour contraindre les tenanciers récalcitrants à honorer leur dette. Tout d'abord, force est de reconnaître que les délais de paiement imposés par la justice sont extrêmement précis et généralement très brefs, sans doute pour éviter de permettre aux débiteurs de se dérober une nouvelle fois. Ainsi, la veuve de Gervaise Proces ne dispose-t-elle que de huit jours pour rembourser les arrérages des années 1413 à 1420, tout comme Colin Cormier qui, dans le même délai, doit pour sa part verser 12 livres aux bénédictines ${ }^{36}$. Cependant, les tenanciers sont le plus souvent sommés de payer dans un délai de deux mois en moyenne. Il est à noter, de plus, que nombreuses sont les personnes qui, se trouvant dans l'incapacité pure et simple de payer la somme exigée par le Ronceray, choisissent de résilier leur bail, avec l'accord des religieuses, ou leur " transportent " un bien ou une rente. Ainsi, Jeanne d'Ancenis abandonne-t-elle aux moniales ses deux maisons, courtils et appartenances rue de la Couldre ${ }^{37}$. À l'instar de cette dernière, Guillaume Béroul cède à l'abbaye ses " deux herbergements avecques courtils, appartenances et dépendances ", à côté du château de Malemort, afin d'être quitte de tout devoir ou rente envers celle-ci ${ }^{38}$. Le dernier moyen de pression dont peuvent user les religieuses du Ronceray ou leurs officiers est la menace de saisie. En signant un nouveau bail en 1486, Pierre Bouchet s'engage à verser un cens de 360 deniers à la dame de la Chambre ${ }^{39}$. Il est à noter qu'Yvonne d'Andigné, alors à cette charge, accepte pour sa part de réduire le montant

35. Prieuré de l'ordre de Grandmont (Maine-et-Loire, arr. Angers, cant. Angers-NordOuest, comm. Avrillé), Arch. dép. de Maine-et-Loire, 254 H 512.

36. Arch. dép. de Maine-et-Loire, 254 H 514.

37. Arch. dép. de Maine-et-Loire, 254 H 479.

38. Arch. dép. de Maine-et-Loire, 254 H 506.

39. Religieuse en charge de la gestion des biens matériels du monastère. Elle est élue par le chapitre. 
initial du cens, dont la somme demeure inconnue. En échange de cette concession, une clause de garantie est ajoutée au bail. Il apparaît ainsi que si le nouveau tenancier ne paie pas les 360 deniers prévus pendant trois ans, le monastère pourra se saisir de tous ses biens ${ }^{40}$. Si le recours à la saisie n'intervient qu'en dernier lieu dans des affaires d'arrérages, les religieuses disposent cependant d'un autre biais leur permettant de récupérer des biens sans que le tenancier ait fait défaut de payer. On parle dans ce cas de " retrait féodal ". Cette pratique est attestée à deux reprises dans les sources de l'abbaye. Il s'agit, selon François-Olivier Touati ${ }^{41}$, de la reprise d'un fief que le vassal souhaite vendre à un acquéreur que le seigneur ne veut pas avoir comme vassal. Elle s'accompagne dès lors d'un dédommagement. La question se pose ainsi en 1470 pour Pierre Le Roy qui possède alors une rente de 720 deniers sur une maison rue de la Tannerie. L'identité de la personne à laquelle il se proposait de la céder n'est pas mentionnée dans l'acte. Seul le montant de la transaction nous est connu. Pierre Le Roy obtient en effet des religieuses 46 écus d'or, 18 sous et 4 deniers en échange de sa rente.

Dès lors, et malgré les indéniables difficultés de recouvrement auxquelles peuvent être confrontés les receveurs du Ronceray, l'abbaye, à l'instar des autres établissements ecclésiastiques ainsi que des seigneurs laïcs, dispose d'une importante protection juridique dont l'efficacité est indubitablement renforcée par une remarquable précision, tenue et conservation des archives fiscales.

\section{Qui paie? Répartition sociale des tenanciers}

Un premier constat ne peut manquer de s'imposer à la lecture du tableau illustrant la répartition sociale des tenanciers : la très forte proportion d'inconnus. En effet, seuls 30 à $45 \%$ des personnes mentionnées dans les censiers sont précisément identifiées. Ceci n'est pas propre au cas du Ronceray. Il s'avère en effet très difficile de mener une étude sociale à partir de sources médiévales où les données juridiques et fiscales l'emportent largement sur les aspects proprement sociaux. En outre, les sources conservées sont en nombre limité, ce qui ne facilite pas les recoupements. Dans ce contexte, les censiers du Ronceray se trouvent être, toute proportion gardée, des sources riches et précieuses pour l'époque au niveau social; surtout ceux de 1386 et 1460, où les qualités de $39 \%$ et $45 \%$ des tenanciers sont respectivement indiquées. En ce qui concerne les chiffres du censier de 1424-1426, dont la fiabilité est sujette à caution, moins de 30 \% des qualités des tenanciers nous sont connues. Aussi ne fournissons-nous le tableau obtenu grâce aux données de ce censier qu'à titre purement indicatif (Tableau 3).

40. Arch. dép. de Maine-et-Loire, 254 H 527, pièce 68.

41. TouATI, François-Olivier, Vocabulaire historique du Moyen Âge, Paris, 1995, p. 191. 
Vivre au-delà des ponts à Angers (1380-1499)

Tableau 3 - Répartition sociale des personnes assujetties à un cens

\begin{tabular}{|l|c|c|c|}
\hline & en 1385-1386 & en 1424-1426 & en 1460-1486 \\
\hline Religieux & 11 & 8 & 7 \\
Religieux de la Trinité & 6 & 7 & 6 \\
Seigneurs & 3 & 2 & 5 \\
Gens de justice & - & - & 2 \\
Veuves & 10 & 8 & 9 \\
Artisans & 9 & 4 & 16 \\
Inconnus & 61 & 71 & 55 \\
\hline
\end{tabular}

Certaines constantes se dégagent nettement. Il apparaît tout d'abord que la population des tenanciers identifiés se répartit en quatre grands groupes : les ecclésiastiques (réguliers ou séculiers), les seigneurs ${ }^{42}$, les veuves et les artisans. Il est frappant de constater que la part des trois premiers groupes n'évolue quasiment pas en cent ans. En revanche, celle des artisans passe de $9 \%$ à $16 \%$. Une question se pose alors. Leur part dans la population des tenanciers a-t-elle réellement augmenté ou les $7 \%$ en plus sont-ils à mettre en lien direct avec les $6 \%$ supplémentaires de personnes identifiées en 1460 par rapport à 1386? Autrement dit, le pourcentage des artisans détenteurs d'un bien sur le fief du Ronceray en 1386 était-il sensiblement le même qu'en 1460; 7 \% d'entre eux étant classés parmi les inconnus à la fin du XIV ${ }^{\mathrm{e}}$ siècle? Toujours est-il que ce groupe occupe une place de choix au sein des tenanciers de la Doutre.

Loin d'être homogènes, les quatre grandes catégories que nous avons distinguées peuvent à leur tour - pour certaines d'entre elles du moins être divisées en sous-groupes. Ainsi les artisans se répartissent-ils en six grandes catégories : les métiers de l'alimentation, du cuir, du bâtiment, du drap, des métaux ainsi que les marchands. De même, les ecclésiastiques forment un groupe très hétérogène qui abrite en son sein des desservants de la Trinité (chanoines et chapelains), des abbayes ${ }^{43}$, des aumôneries et des ordres mendiants ${ }^{44}$, des prieurés ${ }^{45}$, des confréries ${ }^{46}$ ou encore des ecclésiastiques en tant que personnes privées.

42. Nous entendons ici par seigneurs les personnes dont le nom est accompagné dans les documents par la mention "seigneur de... " ou " dame de..." .

43. Abbayes de Bellebranche (Mayenne, arr. Château-Gontier, cant. Grez-en-Bouère), de Clairmont (Mayenne, arr. Laval, cant. Laval), de Fontaine-Daniel (Mayenne, arr. Mayenne, cant. Mayenne-Ouest, comm. Saint-Georges-Buttavent), de la Melleray (LoireAtlantique, arr. Châteaubriand, cant. Moisdon-la-Rivière, comm. Meilleraye-en-Bretagne), de Saint-Georges-sur-Loire (Maine-et-Loire, arr. Angers, cant. Saint-Georges-sur-Loire), de Saint-Nicolas (Maine-et-Loire, Angers)

44. Hôpital Saint-Jean-l'Evangéliste, aumônerie de Saint-Laurent, aumônerie Fils-dePrêtre, aumônerie de Saint-Etienne, couvent des Augustins. Tous ces établissements sont situés dans le quartier de la Doutre à Angers.

45. La Haie-aux-Bonshommes (Maine-et-Loire, arr. Angers, cant. Angers-Nord-Ouest, comm. Avrillé), Juvardeil (Maine-et-Loire, arr. Segré, cant. Châteauneuf-sur-Sarthe), la Papillaie (Maine-et-Loire, Angers), Saintes-Gemmes (Maine-et-Loire, arr. Angers, cant. Les Ponts-de-Cé), Seiches (Maine-et-Loire, arr. Angers, cant. Seiches-sur-le-Loir).

46. Confréries du Saint-Esprit, des Quinze-Vingt Aveugles de Paris. 
Il semble enfin important de s'arrêter quelques instants sur le cas des veuves. En effet, contrairement à ce que l'on serait tenté de croire, les femmes, et notamment les veuves, disposent d'un certain nombre de droits à la fin du Moyen Âge. Plusieurs formules introduites dans les baux de cette époque illustrent parfaitement cet état de fait. Ainsi, lorsqu'un homme et sa femme se portent acquéreurs d'un bail, il n'est pas rare de rencontrer l'expression " ladicte femme suffisamment auttorisée de sondit mari ${ }^{47}$ ". Cette formule est d'ailleurs généralement accompagnée, à la fin de l'acte, de la référence " à touz autres droiz faiz et introduiz en faveur des femmes ${ }^{48}$ ". Ces dernières bénéficient de fait d'une protection juridique importante, notamment lors des successions. Il apparaît ainsi que nombre d'entre elles héritent seules des biens de leur père ou de leur époux ${ }^{49}$. Elles peuvent cependant être tenues d'en partager la jouissance avec leurs enfants, comme en témoigne la mention récurrente dans les censiers : " La femme et hers feu... " Par ailleurs, en cas de remariage - pratique répandue au $\mathrm{XV}^{\mathrm{e}}$ siècle - les veuves transmettent les biens de leur défunt mari à leur nouvel époux, ainsi que les engagements contractés par le premier. Ainsi, en épousant la veuve de Pierre de La Chabocelaye, Jean Chappelain se trouve dans l'obligation de verser aux religieuses du Ronceray les 4 livres de cens que Pierre de La Chabocelaye avait accepté de payer chaque année pour ses biens rue de la Coudre ${ }^{50}$. Toutefois, les femmes agissent rarement seules. En effet, si elles occupent une place non négligeable dans toutes les transactions que nous venons d'évoquer, celles-ci sont cependant réalisées par leurs époux ou, dans le cas des veuves, par des procureurs ${ }^{51}$.

Le quartier de la Doutre apparaît donc, à la fin du Moyen Âge, comme une zone très dynamique de la ville d'Angers de par son activité commerciale et la présence des bénédictines du Ronceray. Son organisation topographique de même que les caractéristiques sociales de sa population nous sont bien connues pour l'époque du fait de la richesse des sources fiscales de l'abbaye. Ainsi, malgré les insuffisances que nous avons évoquées, censiers et baux constituent des mines de renseignements sur les sociétés médiévales. Généralement bien conservés et détaillés, ils se prêtent parfaitement à des études sérielles qui mettent en exergue des évolutions - celles des fiefs, des rapports sociaux, de l'activité économique - et offrent dès lors une vision dynamique de la société et des rapports qui sont les siens.

47. Arch. dép. de Maine-et-Loire, 254 H 34.

48. Idem.

49. En 1420, Colette, fille de Robin Chesdon, hérite des biens que son père tenait rue Corne-de-Cerf (Arch. dép. de Maine-et-Loire, 254 H 478). De même, en 1456, Jeanne, veuve de Jean Chappelain dispose à sa guise des biens qu'elle tenait de son mari sans que ses héritiers s'y opposent (Arch. dép. de Maine-et-Loire, 254 H 478)

50. Arch. dép. de Maine-et-Loire, 254 H 479.

51. Arch. dép. de Maine-et-Loire, 254 H 514. 


\section{Annexe - Extraits du censier de l'abbaye du Ronceray d'Angers pour les années 1385 et 1386}

[Original, XIVe siècle, parchemin : Bibl. mun. d'Angers, ms. 852 (764)]

La transcription ci-après est celle des premiers articles du censier de l'abbaye du Ronceray de 1385-1386 ${ }^{52}$ concernant les biens situés sur le fief de cette dernière dans la seule ville d'Angers ${ }^{53}$. L'introduction de ce livre foncier souligne l'organisation et les finalités de la rédaction d'un tel document. La structure originale de ce type de sources apparaît ici clairement. Il est à noter par ailleurs qu'afin de rendre ce censier aussi accessible que possible, la ponctuation, les majuscules et l'orthographe des mots abrégés ont, comme le veut l'usage, été rétablis selon les règles actuelles. Seules font défaut les notes marginales introduites ultérieurement.

\section{$\left[\mathrm{f}^{\circ} 1\right]$}

Ce sont les cens et rentes madamme l'abbasse du moustier de Noustre Damme d'Angiers renduz à Angiers par chascun an au jour de Noël et receuz par moy Hervé Le Clerc, prestre, censsier de très noble et puissante damme madamme Ysabeau de Ventadour, à present abbasse dudit moustier, ledit jour de Noël l'an mil trois cens quatre vings et cinq. Et sont merchez ceulx qui ont paié pour celui an par $A$, et pour les années ensuivans par les autres lettres de $A, B, C$, par ordre en un autre livre de parchemin duquel cest present livre est extrait.

PREMIEREMENT. En la rue des la Porte du chasteau de l'abbeie en allant sur les Ponts d'Angiers.

JEHAN Le Filleurs, pour une maison joignant à la maison que tint derraniement Jehan Le Barbier, que tient à present Jehan Seguin d'une part, et la maison à la femme et hoirs de feu Thomas Poulain d'autre part, et fut Guillaume Guion et Tenneguy Le Taillendier. Et ne souloit devoir par an que XX s. Et la partie de ladicte maison qui povoit appartenir aux hoirs dudit Tenneguy fut acquise d'eulx l'an mil CCC IIII ${ }^{X X}$. Et fut le tout baillé audit Filleur pour paier par chascun an à cest terme en oultre autant à la Saint-Jehan Baptiste

$X X X s$.

ITEM ledit Filleur, pour un appentiz en la Pasticerie, sis entre la maison à la femme et hoirs dudit feu Thomas Poulain d'une part, et la maison Henry Laguillier, que tient à present Florion Pasticier, d'autre part. Ouquel appentiz est le four à pastez, et le tindrent Guion Naslin et Huet Chauvau; doit à cest terme en oultre autant à la Saint-Jehan Baptiste

$$
X V s \text {. }
$$

JEHAN Seguin, pour la maison que tint derraniement Jehan Le Barbier, sise devant la Porte au pain, joignant d'une part, à la maison Jehan Le Filleurs et d'autre part faisant le coign de la Porte du chasteau de l'abbeie. Et souloit devoir à cest terme et autant à la Saint-Jehan Baptiste XX s. Et elle chaist en main de ladicte abbeie et ne peut estre baillée pour paier à cest terme et autant à la Saint-Jehan Baptiste que à la somme de

52. Le censier de 1385-1386 a fait l'objet d'une transcription intégrale dans mon mémoire de maîtrise : MÉRAND, Anne-Claire, L'abbaye du Ronceray..., op. cit., p. 127 à 259.

53. Le sujet de mon mémoire de maîtrise portant exclusivement sur la ville elle-même, j'ai choisi de ne pas faire apparaître les articles concernant les biens des religieuses « hors la ville". 
LA femme et hoirs de feu Thomas Poulain, pour doux maisons en la Pasticerie, devant la Porte au pain, sises entre la maison où demoure Jehan Le Filleurs d'une part, et l'appentiz où est le four à pastez dudit Filleurs d'autre part; lesquelles maisons furent Jourdain Le Maczon; doivent à cest terme en oultre autant à la Saint-Jehan Baptiste XIII $s$.

ITEM ladicte femme et hoirs dudit feu Thomas Poulain, pour une maison en rue Leonnaise où demourierent Rambaut et Guillaume Doisseau, sise entre la maison Perrot Horeau d'une part, et la maison Guiommar de Gouzillon d'autre part, aboutant d'un bout au pavement de ladicte rue Leonnaise; doivent à cest terme en oultre autant au terme de la Saint-Jehan Baptiste

Xs. VId.

$\left[\mathrm{f}^{\circ} 1 \mathrm{v}^{\circ}\right]$

HENRY Laguilliers, pelletier, pour la maison où il demeure que il ot à cause de sa mère, joignant d'une part, à la Porte au pain et d'autre part à la maison Jamet Pautonnier, aboutant d'un bout au pavement devant les maisons feu Thomas Poulain et d'autre bout au pavement de la Laitterie; doit à cest terme et autant au terme de la Saint-Jehan Baptiste

ITEM, pour une maison en la Pasticerie où a four à pastez que tint Gillet de Mascon, et la tient à present Florion Pasticier, sise entre la maison feu Raoul Juqueau d'une part, et la maison Jehan Le Filleurs d'autre part; en oultre autant au terme de SaintJehan Baptiste, doit à cest terme de Noël

ITEM, pour cuire pain audit four à pastez qui est en la maison dessusdicte; doit à cest terme de Noël, en oultre autant à la Saint-Jehan Baptiste

VII s. VId.

ITEM, pour ladicte maison où est ledit four à pastez qui souloient estre paiez à damme Aliennour de Poilloueille et doit autant à la Saint-Jehan Baptiste

ITEM, pour un estal de la Porte au pain, près son huis devers la Pasticerie, baillé de nouvel et autant à la Saint-Jehan Baptiste

LA femme et hoirs de feu Raoul Juqueau, pour une maison en la Pasticerie, sise entre la maison Henry Laguillier que tient Florion, d'une part, et la maison Julien de Clermont d'autre part; et doit autant à la Saint-Jehan Baptiste

$$
\text { XII s. VId. }
$$

JULIEN de Clermont, pour une maison en la Pasticerie, que tint feu Guillaume Godart, et y a four à pastez, sise entre la maison feu Mace Le Pasticier d'une part, et la maison feu Raoul Juqueau d'autre part, et aboutant au pavement de ladicte Pasticerie; doit à cest terme et autant à la Saint-Jehan Baptiste

XIII s. IX d.

LA femme de feu Guillaume Godart, pour la moitié d'une maison [...] qui fut Juliot Le Corduonnier dont Guillaume Rousseau tient l'autre moitié à cause de sa femme, fille de Gervaise Poulart, sise ladicte maison entre la maison Jehan Bricet, une venelle entre deux, d'une part, et la maison Estienne Langlais, la partie dudit Rousseau entre deux, d'autre part; et doit autant à la Saint-Jehan Baptiste

III s. IXd.

GUILLAUME Rousseau, à cause de sa femme, pour l'autre moitié de ladicte maison dont ladicte Godarde detient l'autre moitié, le tout soubz un fest, et la tint Gervaise Poulart, sise entre la maison de ladicte Godarde d'une part, et la maison Estienne Langlais d'autre part; et doit autant à la Saint-Jehan Baptiste

III s. IXd. 
$\left[\mathrm{f}^{\circ} 2\right]$

PIERRE Le Roy, pour une maison sise sur la Pettite Arche, entre la maison Geuffroy Forestier d'une part, et la maison Robin Quinemere d'autre part, aboutant du bout devant ladicte Pettite Arche que Thevenin Le Bourgoignon, espicier, bailla ja pieça à l'abbeie que il avoit de rente sur ladicte maison et à la descharge de cent souls de rente que il devoit par chascun an à Pasques et à la me-aoust pour moitié sur son herbergement et appartenances de Guignefole, comme appert par lettres, qui sont deus l'abbeie; doit à cest terme et autant au terme de Saint-Jehan Baptiste

$X X X s$.

LA femme et hoirs de feu Mace Garnier, pasticier, pour leur maison où est le four à pastez en la Pasticerie, devant la Boucherie, sise entre entre la maison Julien de Clermont d'une part, et faisant le coign de ladicte Pasticerie, devers la porte de NoustreDamme d'autre part, aboutant au pavement de ladicte Pasticerie; et doit autant à la Saint-Jehan Baptiste

$X X s$.

ITEM, pour ledit four a pastez et pour l'estau en ladicte maison qui furent baillez audit feu Mace à XV s. à cest terme et autant à la Saint-Jehan Baptiste; de laquelle somme sont à rabatre six deniers, et autant à la Saint-Jehan Baptiste, que ils paient à monseigneur d'Aniou pour une [...] qui fut face dudit estau sur le pavement. Ainsi ne demeure rabatuz les VI deniers dessusditz à cest terme et autant à la Saint-Jehan Baptiste, que XIIII s. VId.

ITEM, sur ladicte maison où est ledit four à pastez que les Augustins baillèrent à leur descharge, qui leur estoient deubz par chascun an et autant à la Saint-Jehan Baptiste VII s. VId.

ITEM, ladicte femme et hoirs, sur ladicte maison que feue damme Aliennour de Poiloeille, jadis religieuse de cest moustier, avoit de rente, de laquelle religieuse l'abbeie a à present l'accion; doivent à cest terme et autant à la Saint-Jehan Baptiste

XII s. VId.

OLIVIER Mahaut, chandelier, pour une maison où il vent les chandelles devant la porte de l'eglize de Noustre-Damme, sise entre la maison Guillaume Pennet d'une part, et faisant le coign devant ladicte porte d'autre, aboutant au pavement devant l'oustel Estienne Langlais; doit à cest terme et autant à la Saint-Jehan Baptiste

$X X V s$.

ESTIENNE Barrier, dit Langlais, pour son herbergement devant la porte Noustre-Damme, joignant d'une part, à la maison Guillaume Guenart, que tint Le Mettaier et feu maistre Henry Auberth et Guillemete sa femme, d'une part, et la maison Guillaume Rousseau, que tint Poulart, d'autre part; doit à cest terme et autant à la Saint-Jehan Baptiste XXIIII $s$.

ITEM ledit Estienne Barrier, pour l'appentiz de la salle de la fontaine, derrière la maison dessusdicte et tenent avecques elle d'une part, et aux murs de la maison feu Jehan Maslin, que tient à present Perrot Boitleve l'ainsné; doit en oultre ce que il bailla par assiete à cest terme et autant à la Saint-Jehan Baptiste

$X s$.

$\left[\mathrm{f}^{\circ} 2 \mathrm{v}^{\circ}\right]$

ITEM ledit Estienne Barrier, pour une maison en rue Leonnaise qui fut [...] Chauvete et Richart Le Maczon, et la tint Jehan Le Gentilhome, et souloit devoir X s. Et pour partie des chouses, tenens avecques ladicte maison, qui furent Robert Bourgine et audit Richart Le Maczon, que tint Henry Frerot, et souloient aussi devoir X s., sise ladicte maison et appartenances entre une autre maison qui est audit Estienne d'une part, et 
la maison de Denise La Lambarde d'autre part, aboutant au pavement de ladicte rue Leonnaise. Et lui fut baillée à Noël l'an mil CCCLXXV pour paier à cest terme et autant à la Saint-Jehan Baptiste

ITEM, pour une autre maison en ladicte rue Leonnaise que tint Perrin Le Maczon, et y demoura Guillaume Barrier, joignant et tenent d'une part, à la maison que tient Perrot Horeau, aboutant au pavement d'icelle rue; doit à cest terme en oultre autant au terme de Saint-Jehan Baptiste

ITEM, pour un courtil au port à la Hoquette que tint Pierre Guerin, sis au long de la riviere de Maienne d'une part, et à la maison feu Jehan Pichart d'autre part; et doit autant à la Saint-Jehan Baptiste

\section{RÉSUMÉ}

Dans les deux derniers siècles du Moyen Âge, l'abbaye féminine du Ronceray s'affirme comme l'un des principaux centres de pouvoir de la ville d'Angers de par la notoriété que lui confèrent l'origine noble de ses bénédictines mais aussi la sacralité de ce haut lieu de dévotion mariale. L'importance des possessions des religieuses et des revenus qui en découlent renforce encore l'influence des moniales dans la cité. Celles-ci dominent de fait près de la moitié de la ville tant au niveau économique que spirituel. Presque exclusivement implantées sur la rive droite de la Maine, les religieuses du Ronceray voient leur liberté d'action renforcée par le relatif isolement géographique que génère le passage de la rivière. Elles n'ont en outre rien à envier aux établissements masculins angevins en ce qui concerne l'ampleur de leurs revenus, leur fief étant des plus lucratifs et rémunérateurs. Aussi, après avoir défini le " grand fief " des bénédictines du Ronceray, nous sommes nous intéressés aux différents revenus issus du temporel urbain de l'abbaye, à leur évolution ainsi qu'à leurs tenanciers entre 1380 et 1499.

\section{ABSTRACT}

During the last two centuries of the Middle Age, the women's abbey of Ronceray became one of the most powerful centers of the city of Angers because of the nobility of the nuns and the sacredness of this place devoted to Mary. The importance of the nuns' possessions and incomes still strengthened their influence in the city. Indeed, they dominated almost half of the city economically as well as spiritually. Nearly exclusively established on the right shore of the river Maine, the nuns of Ronceray enjoyed a liberty of action increased by the relative geographic isolation generated by the river. Moreover they had no reason to envy the men's monasteries' incomes for their fief is one of the most lucrative and paying. So, after having defined the "grand fief" of the nuns of the Ronceray, we'll analyse the different incomes of the abbey's urban fief, their evolution and their owners between 1380 and 1499. 